Short Report

\title{
Beyond CPRA: Identifying Sensitized Kidney Candidates with Markedly Low Access to Deceased Donor Transplantation by Granular CPRA and Blood Type
}

Angela Q Maldonado ${ }^{1,{ }^{+},{ }^{*}}$, Kristoffer Sjöholm ${ }^{1,{ }^{\dagger}}$, Joshua Lee ${ }^{1,{ }^{+},{ }^{\ddagger}}$, Håkan Olsson ${ }^{1,{ }^{+}}$, Christian Kjellman ${ }^{1,{ }^{+}}$, Darren E Stewart ${ }^{2,+}$

1. Hansa Biopharma AB, Scheelevägen 22, 22363 Lund, Sweden; E-Mails:

angela.maldonado@hansabiopharma.com; kristoffer.sjoholm@hansabiopharma.com;

joshua.lee@hansbiopharma.com; hakan.olsson@hansabiopharma.com;

christian.kjellman@hansabiopharma.com

2. United Network for Organ Sharing, $700 \mathrm{~N} 4^{\text {th }}$ St, Richmond, VA 23219, US; E-Mail: darren.stewart@unos.org

‡ Current Affiliation: Immucor, Inc.

$\dagger$ These authors contributed equally to this work.

* Correspondence: Angela Q Maldonado; E-Mail: angela.maldonado@hansabiopharma.com

Academic Editor: Christophe Picard

Special Issue: Donor Specific Antibody

OBM Transplantation

2021, volume 5, issue 2

doi:10.21926/obm.transplant.2102143
Received: April 01, 2021

Accepted: May 24, 2021

Published: June 03,2021

\begin{abstract}
Prioritization in the US Kidney Allocation System (KAS) has led to an improvement in the rates of transplantation in highly sensitized (HS) patients. However, there is a subset of HS patients who are at a disadvantage, despite prioritization under KAS. The purpose of this study was to describe the transplant rate (TR) by calculated panel reactive antibody (CPRA) of HS candidates before and five years post-KAS to characterize their access to deceased donor transplants and quantify the number of HS candidates who are at a marked disadvantage in
\end{abstract}

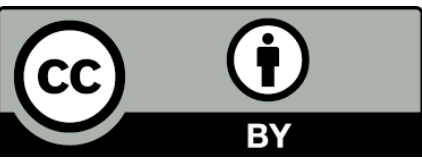

(C) 2021 by the author. This is an open access article distributed under the conditions of the Creative Commons by Attribution License, which permits unrestricted use, distribution, and reproduction in any medium or format, provided the original work is correctly cited. 
accessing kidneys. Using de-identified OPTN data, the number of solitary deceased donor kidney transplants performed (by month) from June 2013 through December 2019 was analyzed for four CPRA groups (95-97\%, 98\%, 99\%, and 100\%), with the CPRA 100\% group further stratified into two subgroups (99.5-99.9\% and $>99.9 \%)$. The impact of the recipient blood group was assessed as an additional factor in TR. Immediately after KAS, TR rose 7-fold from $0.26 \%$ to $1.7 \%$ for the CPRA $99-100 \%$ group as a whole, and 12 -fold and 8 -fold for the CPRA 99.5-99.9\% and CPRA 99.9\%+ groups, respectively. However, the post-KAS mean TR of $0.63 \%$ for CPRA $99.9 \%$ + candidates remained markedly lower than the mean TR of the other sensitized groups: $1.5 \%$ for CPRA 95-97\%, 1.4\% for CPRA 98\%, 2.0\% for CPRA 99\%, and 3.5\% for CPRA 99.5-99.9\%; a statistically significant 6-fold advantage of the CPRA 99.5-99.9\% group over CPRA 99.9\%+ candidates was observed, despite both groups receiving national priority under KAS. Patients with a CPRA of $99.9 \%+$ and blood group B were 2.16, 1.98, and 1.75 times less likely to receive a transplant compared to the same CPRA group with blood groups $A, A B$, and $O$, respectively, despite changes to $K A S$ allocating blood type $A 2$ and $A 2 B$ to recipients with blood group $B$. Although the TR increased sharply for the CPRA 99.9\%+ group with KAS, it remained markedly lower than the average despite national priority.

\section{Keywords}

Highly sensitized; kidney allocation; blood group; CPRA

\section{Introduction}

In December 2014, a revised Kidney Allocation System (KAS) was implemented in the US to achieve several goals, including reducing the disparities in the access to transplantation for certain groups of patients. Policymakers recognized that a gradual rise in the donor pool over time would not be sufficient to ensure equitable access to transplants for some candidates - particularly those who were biologically incompatible with a high percentage of the donors. Instead, these patients would require substantial allocation priority such that when a compatible donor became available, they would be at or near the top of the list. Among the modifications, which strove to balance the ethical principles of allocating a scarce resource, was the goal of reducing differences in transplant access for populations described in the National Organ Transplant Act, including highly sensitized candidates (HS) and minority populations with ABO blood group-related waiting list disparities [1, 2]. To better reflect the biology of the sensitized population, additional priority points were awarded to sensitized patients on a continuous sliding scale to improve organ offers and transplant rates for those with a calculated panel reactive antibody (CPRA) $\geq 98 \%$; CPRA of $98 \%$, 99\%, and $100 \%$ received 24.4, 50.09, and 202.10 points, as well as, local, regional, and national priority, respectively [1]. Additionally, A2 and A2B kidneys were allocated preferentially to medically eligible blood group B recipients to improve the transplant rates for these patients [1, 3].

Analysis of KAS three years post-implementation demonstrated an initial bolus effect of transplantation for HS candidates followed by an overall steady state. Using adjusted negative binomial regression, Jackson et al. had analyzed transplant rates post-KAS and found that compared to the pre-KAS rates, candidates with a CPRA of $98 \%$ had an equivalent TR compared to non-HS 
candidates, whereas candidates with a CPRA of 99\% had a higher rate compared to non-HS candidates. However, candidates with the highest CPRA $(99.9 \%+)$ had a significantly lower TR [4]. This finding was confirmed by Schinstock et al. in an analysis performed approximately four years after the implementation of KAS [5]. Even with the advent of kidney paired donation and living donor desensitization options, only a small fraction of HS patients received transplants through these modalities, and a large proportion of the patients with CPRA 99.9\%+ had been waiting for more than five years, despite the changes in $\operatorname{KAS}[5,6]$.

Patients with blood group $B$ generally are less likely to receive transplants than recipients in other blood groups [7]. With the first report on the allocation of A2/A2B deceased donor kidneys to blood group $B$ recipients, the likelihood of transplantation after two years on the waiting list was only $18.3 \%$ for blood group $B$ candidates, compared to $52.6 \%, 38 \%$, and $22.4 \%$ for candidates with blood groups $A B, A$, and $O$, respectively [8]. After the implementation of the revised $K A S$, there was greater access to transplantation for blood type $B$ candidates who could safely accept a kidney from A2/A2B donors. However, full implementation was hindered by the lack of consensus on acceptable anti-A2 titers [9]. Thus, highly sensitized blood group B patients may constitute a subgroup with compounded lower access to transplants.

Given the marked heterogeneity of patients deemed highly sensitized, especially patients in the CPRA 99.9\%+ group, the purpose of this report was to 1) describe the transplant rate (TR) by CPRA of HS candidates before KAS through five years post-implementation; 2) quantify the volume of sensitized candidates having a persistent and marked disadvantage in the access to transplantation based on CPRA or CPRA in combination with blood type; 3 ) describe the demographics and medical characteristics of these patients.

\section{Materials and Methods}

Using de-identified, IRB exempt data (aggregated by month) from the Organ Procurement and Transplantation Network (OPTN), we performed a retrospective analysis of patients on the kidney transplant waiting list in the United States. The number of patients on the waitlist and the number of transplants performed were tallied at month-end. Each patient was counted once and the highest CPRA listed was used if the patient had multiple listings. Comparator groups were divided into the pre-KAS period, from June 1 (2013) through November 30 (2014), and the post-KAS period, from December 1 (2014) through December 31 (2019). However, since the data were aggregated by month and KAS was implemented on December 4 (2014), three days of the December 2014 postKAS cohort were actually pre-KAS.

Transplant rates (TR) were calculated as the number of solitary deceased donor kidney transplants performed by month, divided by the number of candidates on the waiting list (WL) at the end of the previous month. Rates were calculated overall, including all candidates waiting for a solitary kidney transplant, and stratified by four CPRA groups (95-97\%, 98\%, 99\%, and 100\%). The CPRA, $100 \%$ group, was further divided into two subgroups (99.5-99.9\% and $99.9 \%+)$. The monthly TR was also expressed as pre-and post-KAS means and examined temporally. The CPRA 99\%+ group was examined in more detail as discrete CPRA groups as follows: $99.5 \%$ to $<99.6 \%, 99.6 \%$ to $<99.7 \%$, $99.7 \%$ to $<99.8 \%, 99.8 \%$ to $<99.9 \%, 99.9 \%$ to $<99.95 \%, 99.95 \%$ to $<99.975 \%, 99.975 \%$ to $<99.99 \%$, and $99.99 \%$ to $100 \%$. All groups were further analyzed according to candidate blood type $(A, B, A B$, and O). All data were de-identified (IRB exempt). 


\subsection{Statistical Analysis}

A chi-squared contingency table test was used for statistical analysis of demographic data, and Student's t-test was used for TR data. Trendlines were fitted using linear regressions with 95\% confidence intervals. All statistical analyses were performed in R version 3.5.2 (R Core Team, 2018). Differences between groups were considered to be statistically significant at $p<0.05$.

\section{Results}

Immediately after the implementation of the revised KAS, TR rose 7 -fold from $0.26 \%$ to $1.7 \%$ for the CPRA 99-100\% group as a whole and 12-fold and 8-fold for CPRA 99.5-99.9\% and 99.9\%+ groups, respectively (Figure 1). However, the post-KAS mean TR of $0.63 \%$ for CPRA $99.9 \%+$ candidates remained markedly lower compared to that in the other sensitized groups; $1.5 \%$ for CPRA 95-97\%, 1.4\% for CPRA 98\%, 2.0\% for CPRA 99\%, and 3.5\% for CPRA 99.5-99.9\%. There was a 6-fold (statistically significant) advantage for the latter group over CPRA $99.9 \%+$ candidates, despite both groups receiving national priority under KAS (Figure 1). This is especially evident beginning with the CPRA $\geq 99.95 \%$ group (Figure 2 ). Figure 1 also shows that the TR increased for all CPRA groups except for the highly sensitized group $(99.9 \%+)$, suggesting that only this group had not benefitted from the steady rise in donation over the past decade.

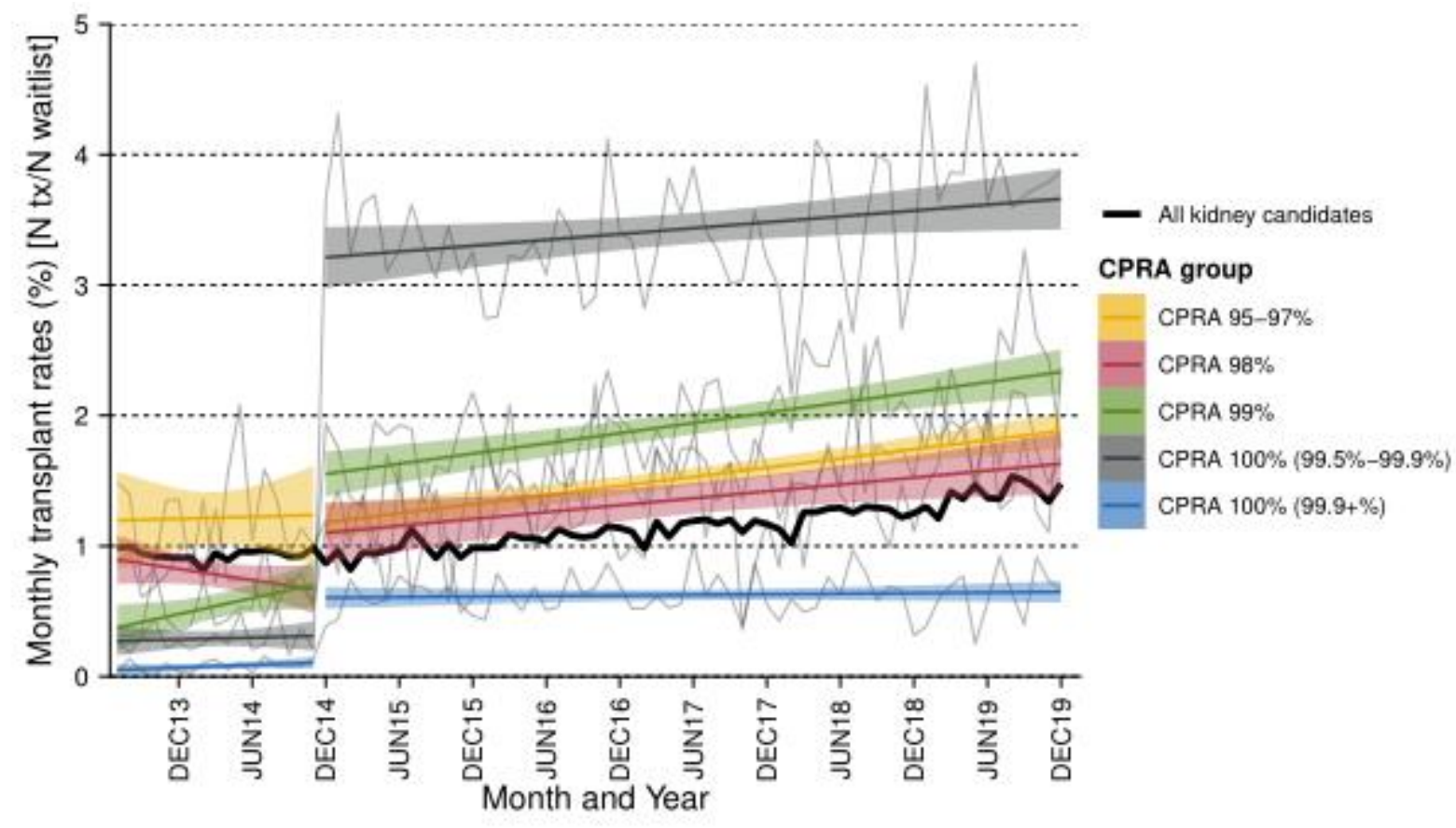

Figure 1 Pre and post-KAS trends in the monthly deceased donor transplant rate (TR). Shown are the individual monthly TR as thin grey lines, with overlayed linear trendline; colors represent the different CPRA groups. The trendlines are shown for the pre and post-KAS periods with the $95 \%$ confidence interval. TR for all kidney candidates (regardless of sensitization) is shown as a thick black line. 


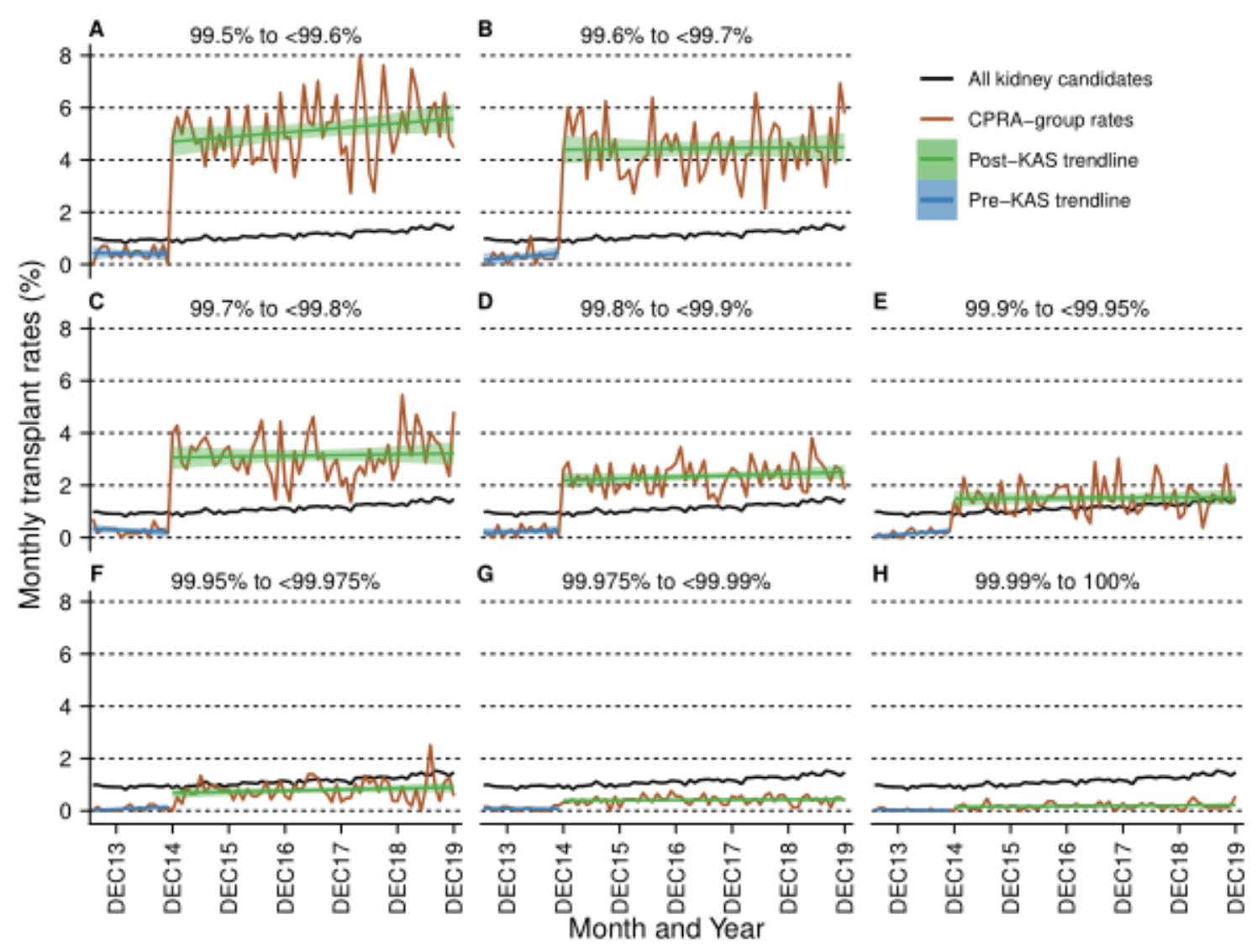

Figure 2 Pre and post-KAS trends in the monthly deceased donor transplant rate (TR) for the CPRA 99.5-100\% groups. Shown are the individual monthly TR for CPRA-groups as brown lines, with overlayed linear trendline for the pre-and post-KAS periods with the 95\% confidence interval. TR for all kidney candidates (regardless of sensitization) is shown as a black line. A) CPRA $99.5 \%$ to $<99.6 \%$, B) CPRA $99.6 \%$ to $<99.7 \%$, C) CPRA $99.7 \%$ to $<99.8 \%$, D) CPRA $99.8 \%$ to $<99.9 \%$, E) CPRA $99.9 \%$ to $<99.95 \%$, F) CPRA $99.95 \%$ to $<99.975 \%$, G) CPRA $99.975 \%$ to $<99.99 \%$, and H) CPRA $99.99 \%$ to $100 \%$.

The number of kidney transplant candidates on the deceased donor transplant waitlist at the end of each month compared to the number of transplants is depicted in Figure 3. Beginning with CPRA 99.5\% and up to CPRA $100 \%$, the number on the waitlist for each incremental CPRA breakpoint steadily increased, and the CPRA $99.99 \%+$ patients accounted for more than $20 \%(n=869)$ of the total waitlist of the CPRA $100 \%$ group. 


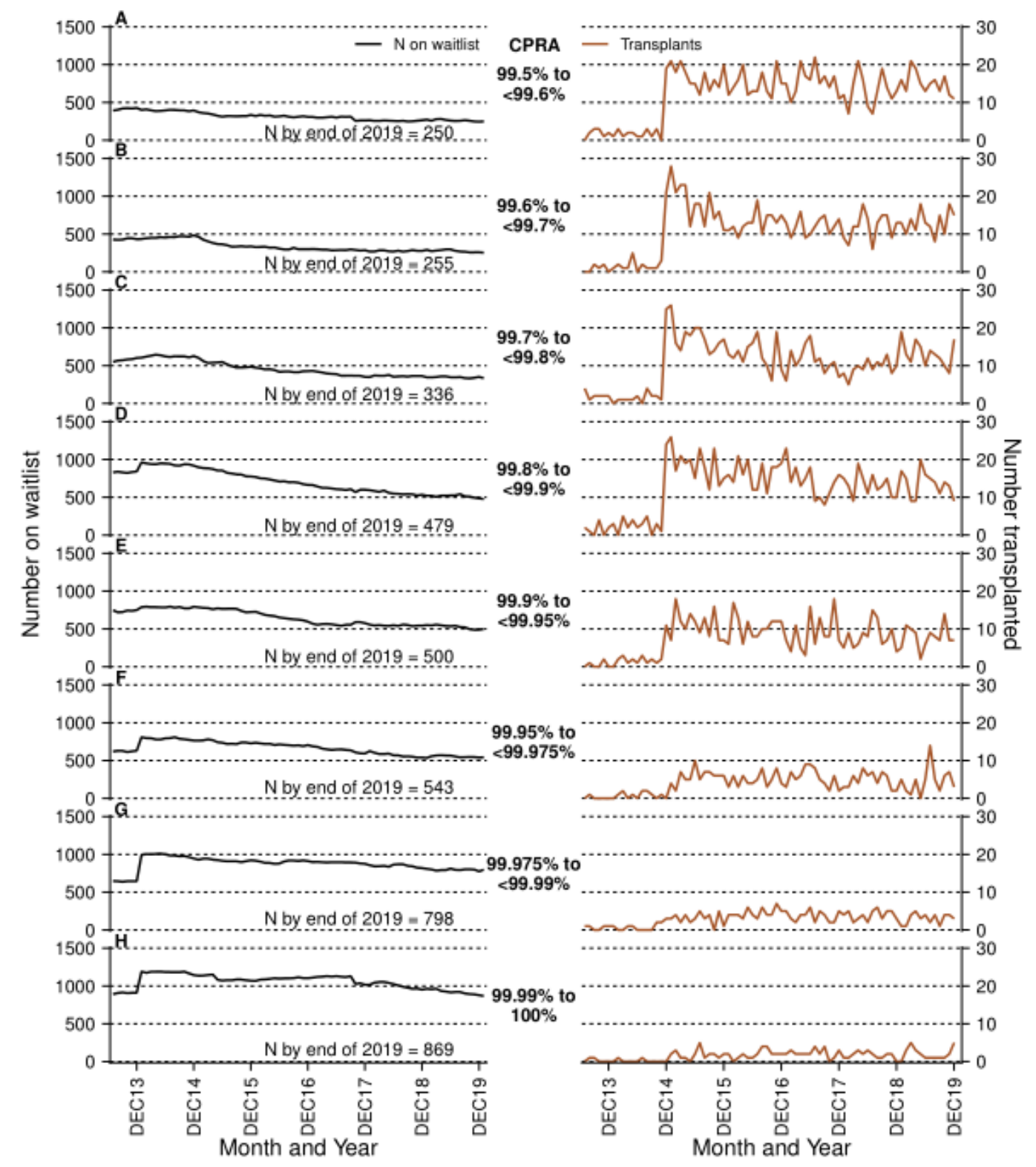

Figure 3 The number of kidney transplant candidates on the deceased donor transplant waitlist at the end of each month (left panel), and the number of monthly transplants (right panel) for the CPRA 99.5-100\% groups. A) CPRA $99.5 \%$ to $<99.6 \%$, B) CPRA $99.6 \%$ to $<99.7 \%$, C) CPRA $99.7 \%$ to $<99.8 \%$, D) CPRA $99.8 \%$ to $<99.9 \%$, E) CPRA $99.9 \%$ to $<99.95 \%$, F) CPRA $99.95 \%$ to $<99.975 \%$, G) CPRA $99.975 \%$ to $<99.99 \%$, and H) CPRA $99.99 \%$ to $100 \%$.

When evaluating the mean monthly TR post-KAS of the CPRA groups ranging from $0-100 \%$ and stratified by blood group, the blood groups $B$ and $O$ demonstrated comparable TRs at low levels of sensitization but started diverging at approximately CPRA $\geq 70 \%$ (Figure 4 ). We defined markedly low transplant access as $75 \%$ of the mean TR of all transplants. TRs were markedly below average for blood type B patients with CPRA 70-79\%, 96-98\%, and 99.25-99.5\%. Starting from CPRA 99.9\%+, patients with blood group $B$ were the most disadvantaged with the lowest TR, while the TR for blood groups $A, A B$, and $O$ fell sharply below average above CPRA 99.95\%. Patients with a CPRA 99.9\%+ and blood group $B$ were $2.16,1.98$, and 1.75 times less likely to receive a transplant compared to 
the same CPRA groups with blood groups $A, A B$, and $O$, respectively, despite the changes to KAS for allocating blood type $A 2$ and $A 2 B$ to recipients in blood group $B$ candidates (Figure 4).

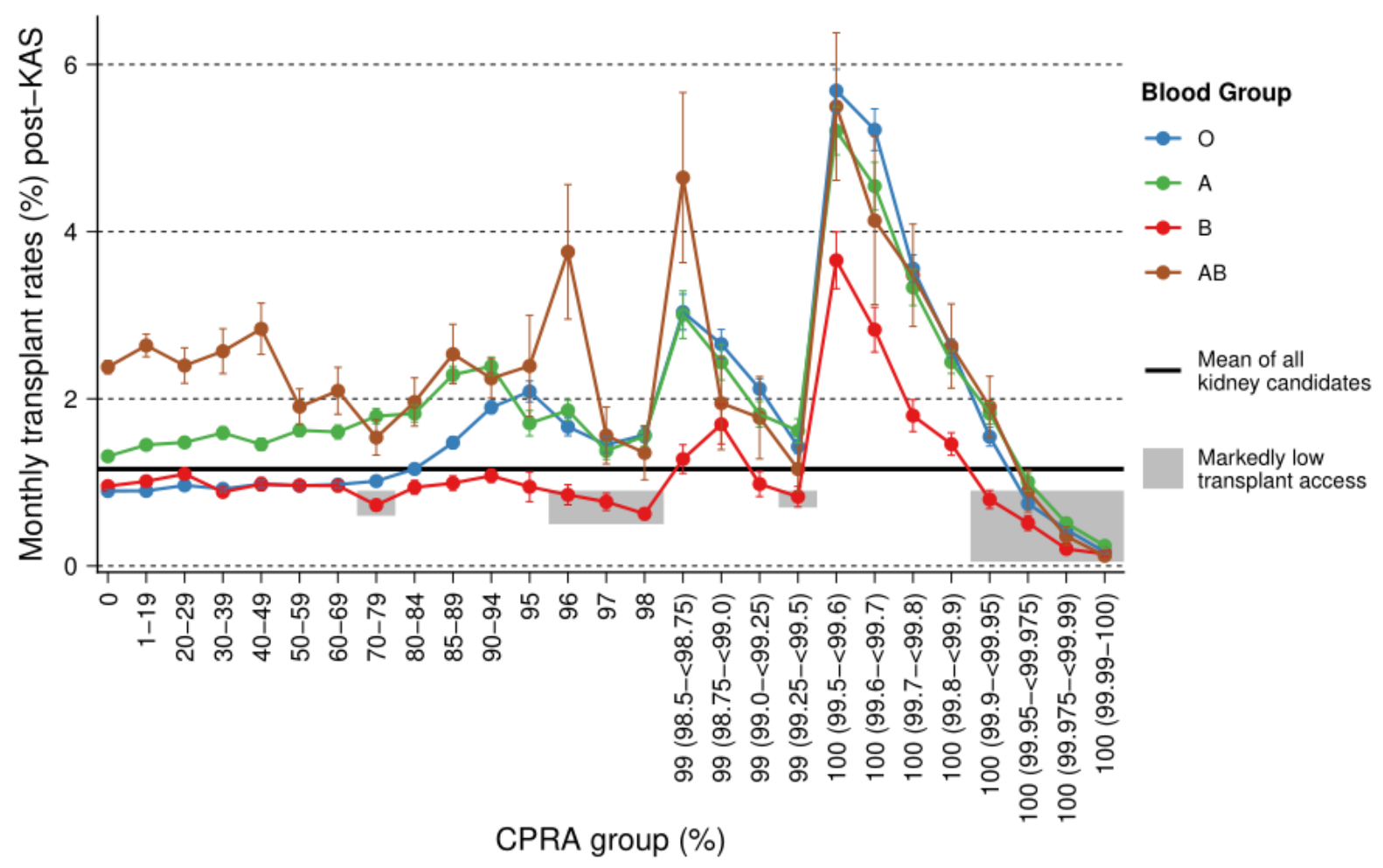

Figure 4 Mean monthly deceased donor transplant rate (TR) post-KAS for blood groups and CPRA-groups ranging from $0 \%$ (non-sensitized) to $100 \%$. Means of blood groups are shown as points connected by lines; colors represent different blood groups. Error bars represent the standard error of the mean. Mean TR for all kidney candidates (regardless of sensitization and blood group) is shown as a horizontal black line. Candidates with markedly low transplant access (i.e., < 75\% TR) are placed on a grey background.

By the end of 2019, 2,710 "kidney-alone" candidates on the waiting list had a CPRA of $99.9 \%+$. The demographic and medical profile of these ultra-sensitized candidates differed substantially from other patients. Compared to all kidney candidates, the CPRA 99.9\%+ candidates were more often younger, female, African-American ( $A A)$, repeat transplant candidates, and non-diabetic. Although just $31.9 \%$ of all kidney candidates were AA, over half of the CPRA $99.9 \%+$ were AA. Compared to the median waiting time of 1.84 years for all candidates still waiting, the median waiting time for the CPRA $99.9 \%+$ candidates was 3.6 years; $26 \%$ had already waited $5-10$ years, while $10 \%$ had been on the list for over 10 years (Table 1 ). The median waitlist time for the CPRA $99.9 \%+$ candidates was twice that of all candidates. Additionally, the proportions of the CPRA $99.9 \%+$ candidates who had waited for $5-10$ years, and more than 10 years, were two times and six times higher than the other candidates, respectively. 
Table 1 Demographic characteristics of candidates on the waitlist at the end of 2019.

\begin{tabular}{|c|c|c|c|c|c|c|c|}
\hline Parameter & Group & All candidates & $<95$ & $95-<99.5$ & $99.5-<99.9$ & $99.9+$ & $p$-value \\
\hline \multirow{5}{*}{ Age } & $0-17$ & $1017(1.1 \%)$ & 950 (1.1\%) & $25(0.7 \%)$ & $21(1.6 \%)$ & $21(0.8 \%)$ & \multirow{5}{*}{$<0.001$} \\
\hline & $18-34$ & $7773(8.4 \%)$ & $6848(8 \%)$ & 411 (11.9\%) & $167(12.7 \%)$ & $347(12.8 \%)$ & \\
\hline & $35-49$ & $21236(22.9 \%)$ & $18827(22.1 \%)$ & $1071(31.1 \%)$ & $423(32 \%)$ & $915(33.8 \%)$ & \\
\hline & $50-64$ & 39960 (43.1\%) & $36886(43.2 \%)$ & $1406(40.8 \%)$ & $538(40.8 \%)$ & $1130(41.7 \%)$ & \\
\hline & $65+$ & $22786(24.6 \%)$ & $21787(25.5 \%)$ & $531(15.4 \%)$ & $171(13 \%)$ & $297(11 \%)$ & \\
\hline \multirow{2}{*}{ Gender } & Female & $35434(38.2 \%)$ & $30838(36.2 \%)$ & $2324(67.5 \%)$ & 780 (59.1\%) & $1492(55.1 \%)$ & \multirow{2}{*}{$<0.001$} \\
\hline & Male & $57338(61.8 \%)$ & $54460(63.8 \%)$ & $1120(32.5 \%)$ & $540(40.9 \%)$ & $1218(44.9 \%)$ & \\
\hline \multirow{6}{*}{ Ethnicity } & White & 32666 (35.2\%) & 30615 (35.9\%) & $1062(30.8 \%)$ & $342(25.9 \%)$ & 647 (23.9\%) & \multirow{6}{*}{$<0.001$} \\
\hline & Black & 29609 (31.9\%) & $26121(30.6 \%)$ & $1446(42 \%)$ & $629(47.7 \%)$ & $1413(52.1 \%)$ & \\
\hline & Hispanic & $19753(21.3 \%)$ & $18553(21.8 \%)$ & $606(17.6 \%)$ & $219(16.6 \%)$ & $375(13.8 \%)$ & \\
\hline & Asian & $8382(9 \%)$ & 7845 (9.2\%) & $241(7 \%)$ & $92(7 \%)$ & $204(7.5 \%)$ & \\
\hline & Amer Ind/Alaska Native & $844(0.9 \%)$ & 786 (0.9\%) & $30(0.9 \%)$ & $6(0.5 \%)$ & $22(0.8 \%)$ & \\
\hline & $\begin{array}{l}\text { Native Hawaiian/other } \\
\text { Pacific Islander }\end{array}$ & $566(0.6 \%)$ & $520(0.6 \%)$ & $19(0.6 \%)$ & $11(0.8 \%)$ & $16(0.6 \%)$ & \\
\hline \multirow{9}{*}{ Blood group } & Multiracial & 952 (1\%) & 858 (1\%) & $40(1.2 \%)$ & $21(1.6 \%)$ & $33(1.2 \%)$ & \multirow{5}{*}{$<0.001$} \\
\hline & $A$ & $25136(27.1 \%)$ & 22975 (26.9\%) & $1006(29.2 \%)$ & 377 (28.6\%) & 778 (28.7\%) & \\
\hline & $A B$ & $2372(2.6 \%)$ & 2099 (2.5\%) & 115 (3.3\%) & $43(3.3 \%)$ & $115(4.2 \%)$ & \\
\hline & B & 15319 (16.5\%) & $13862(16.3 \%)$ & 697 (20.2\%) & $290(22 \%)$ & $470(17.3 \%)$ & \\
\hline & 0 & 49465 (53.3\%) & 45931 (53.8\%) & $1607(46.7 \%)$ & 597 (45.2\%) & $1330(49.1 \%)$ & \\
\hline & $\mathrm{A} 1$ & $406(0.4 \%)$ & $362(0.4 \%)$ & $17(0.5 \%)$ & $13(1 \%)$ & $14(0.5 \%)$ & \multirow{4}{*}{ NA } \\
\hline & $A 1 B$ & $20(0 \%)$ & $18(0 \%)$ & $0(0 \%)$ & $0(0 \%)$ & $2(0.1 \%)$ & \\
\hline & $\mathrm{A} 2$ & $48(0.1 \%)$ & 47 (0.1\%) & $1(0 \%)$ & $0(0 \%)$ & $0(0 \%)$ & \\
\hline & $A 2 B$ & $6(0 \%)$ & $4(0 \%)$ & $1(0 \%)$ & $0(0 \%)$ & $1(0 \%)$ & \\
\hline \multirow{2}{*}{ Previous Tx } & No & $82129(88.5 \%)$ & 78984 (92.6\%) & $1850(53.7 \%)$ & 514 (38.9\%) & 781 (28.8\%) & \multirow{2}{*}{$<0.001$} \\
\hline & Yes & $10643(11.5 \%)$ & $6314(7.4 \%)$ & $1594(46.3 \%)$ & 806 (61.1\%) & $1929(71.2 \%)$ & \\
\hline
\end{tabular}


OBM Transplantation 2021; 5(2), doi:10.21926/obm.transplant.2102143

\begin{tabular}{|c|c|c|c|c|c|c|c|}
\hline \multirow{5}{*}{ Diabetes status } & Not Reported & $207(0.2 \%)$ & $200(0.2 \%)$ & $5(0.1 \%)$ & $0(0 \%)$ & $2(0.1 \%)$ & NA \\
\hline & No & $50498(54.4 \%)$ & 45248 (53\%) & $2274(66 \%)$ & 933 (70.7\%) & 2043 (75.4\%) & \\
\hline & Type I & 2912 (3.1\%) & 2606 (3.1\%) & $144(4.2 \%)$ & $54(4.1 \%)$ & $108(4 \%)$ & $<0.001$ \\
\hline & Type II & 38842 (41.9\%) & 36980 (43.4\%) & 1001 (29.1\%) & $322(24.4 \%)$ & 539 (19.9\%) & \\
\hline & Type Other & $313(0.3 \%)$ & $264(0.3 \%)$ & $20(0.6 \%)$ & $11(0.8 \%)$ & $18(0.7 \%)$ & NA \\
\hline \multirow{6}{*}{ Waiting Time } & Median & 672 days & 652 days & 797 days & 912 days & 1299 days & NA \\
\hline & $<1$ year & 29108 (31.4\%) & $27414(32.1 \%)$ & $946(27.5 \%)$ & $320(24.2 \%)$ & 428 (15.8\%) & \multirow{5}{*}{$<0.001$} \\
\hline & $1-3$ years & 33276 (35.9\%) & 30914 (36.2\%) & $1162(33.7 \%)$ & 427 (32.3\%) & 773 (28.5\%) & \\
\hline & $3-5$ years & $16733(18 \%)$ & 15429 (18.1\%) & $558(16.2 \%)$ & $226(17.1 \%)$ & $520(19.2 \%)$ & \\
\hline & $5-10$ years & 12026 (13\%) & 10423 (12.2\%) & 632 (18.4\%) & 265 (20.1\%) & 706 (26.1\%) & \\
\hline & >10 years & $1629(1.8 \%)$ & $1118(1.3 \%)$ & $146(4.2 \%)$ & $82(6.2 \%)$ & 283 (10.4\%) & \\
\hline
\end{tabular}


In addition to the 470 blood type B candidates with CPRA 99.9\%+ on the list in December 2019, 807 additional blood type B candidates, who were still waiting, had markedly lower access to transplant as shown in Figure 4; 357 with CPRA 70-79\%, 359 with CPRA 96-98\%, and 91 with CPRA 99.25-99.5\%. In total, 3,426 candidates who were still waiting had either CPRA 99.9\%+ or were identified as having markedly lower than average transplant rates associated with the combination of high CPRA and blood type B (Table 2).

Table 2 Transplant candidates waiting on December 31 (2019), categorized by blood group. Highlighted in green: 3,426 candidates still waiting had either CPRA 99.9\%+ or markedly low transplant rates ( $<75 \%$ of mean TR, as highlighted in Figure 4 ) based on the combination of high CPRA and blood type $B$.

\begin{tabular}{|c|c|c|c|c|}
\hline \multirow{2}{*}{ CPRA group (\%) } & \multicolumn{4}{|c|}{ Blood group } \\
\hline & 0 & $A$ & B & $A B$ \\
\hline 0 & 29431 & 15464 & 8641 & 1319 \\
\hline $1-19$ & 6090 & 2855 & 1851 & 271 \\
\hline $20-29$ & 1923 & 974 & 611 & 94 \\
\hline $30-39$ & 1512 & 758 & 446 & 66 \\
\hline $40-49$ & 1072 & 517 & 352 & 47 \\
\hline $50-59$ & 1679 & 749 & 489 & 71 \\
\hline $60-69$ & 1208 & 579 & 413 & 53 \\
\hline 70-79 & 1094 & 507 & 357 & 61 \\
\hline $80-84$ & 591 & 338 & 202 & 42 \\
\hline $85-89$ & 619 & 284 & 206 & 37 \\
\hline $90-94$ & 713 & 360 & 294 & 60 \\
\hline 95 & 167 & 109 & 62 & 5 \\
\hline 96 & 204 & 127 & 88 & 14 \\
\hline 97 & 287 & 175 & 107 & 18 \\
\hline 98 & 384 & 212 & 164 & 28 \\
\hline $99(98.5-<98.75)$ & 108 & 85 & 58 & 3 \\
\hline $99(98.75-<99.0)$ & 117 & 97 & 63 & 10 \\
\hline $99(99.0-<99.25)$ & 140 & 99 & 64 & 15 \\
\hline $99(99.25-<99.5)$ & 200 & 120 & 91 & 23 \\
\hline $100(99.5-<99.6)$ & 113 & 78 & 52 & 7 \\
\hline $100(99.6-<99.7)$ & 114 & 77 & 55 & 9 \\
\hline $100(99.7-<99.8)$ & 153 & 92 & 81 & 10 \\
\hline $100(99.8-<99.9)$ & 217 & 143 & 102 & 17 \\
\hline $100(99.9-<99.95)$ & 257 & 140 & 83 & 20 \\
\hline $100(99.95-<99.975)$ & 263 & 159 & 100 & 21 \\
\hline $100(99.975-<99.99)$ & 386 & 230 & 141 & 41 \\
\hline 100 (99.99-100) & 424 & 263 & 146 & 36 \\
\hline
\end{tabular}




\section{Discussion}

Early post-KAS analyses revealed trends regarding transplant rates across CPRA groups, but extrapolating these trends into the future proved challenging given the bolus effects seen immediately post-implementation. Analyses at the first, second, third, and fourth years post-KAS provided clarity regarding the types of patients who had benefited from the changes introduced and indicated that the system had stabilized over time [5, 6, 10-13]. Ostensibly fueled by the overall rise in donors, TRs steadily increased under KAS for all groups with one notable exception, the highly sensitized group (CPRA 99.9\%+), whose TR remained less than half that of the average candidate. Among HS candidates, the TRs for CPRA 99.5-99.9\% candidates were the highest, implying that national priority was sufficient to overcome this group's immunological barriers to receive transplants. Contrastingly, although the TR of the CPRA 99.9\%+ candidates increased sharply with KAS, five years later, it remained markedly lower than the average, despite national priority. Stratifying this group even further showed that for the CPRA 99.99\%+ candidates, the transplant rate plummeted essentially to zero. The characteristics and demographics of the CPRA 99.9\%+ group remain to be further explored; however, they represent a substantial number of patients.

Given that more than $50 \%$ of the demographic makeup of the CPRA $99.9 \%+$ were AA, with the median waiting time for this group higher than the median waiting time for all candidates, the compounded effect of blood group in addition to sensitization is particularly relevant for this subset of patients. In a report by Martins et al., the authors described the rates of A2/A2B deceased donor transplants pre-and post-KAS [14]. There was a 4.9-fold increase in the likelihood of $A 2$ incompatible deceased donor kidney transplantation compared to pre-KAS, although the increase was mainly attributed to Caucasian patients as there was no difference in the transplant rate among the minority populations [14]. Although sensitization and blood group are often considered individual factors for a decrease in access to transplantation, AA patients in our analysis demonstrated a substantially low TR due to both factors. Thus, future interventions aimed at improving access to transplants for minorities should be prioritized.

As of December 31 (2019), there were nearly 3,000 CPRA 99.9\%+ patients on the waiting list. When considering the HLA and the blood group immunological barriers to transplantation, our analysis revealed that an additional 700 (approx.) highly sensitized candidates with blood type B also had markedly lower than average transplant rates. Our analysis indicated that the number of patients removed from this group was not solely because they had received transplants and might represent a large number of patients who were too sick or ineligible for transplantation and/or died waiting for a transplantover that extended time period. Although 3,000 patients may seem to be a fraction of the total number on the kidney transplant waitlist, it is a significant number of patients when compared to that of the lung transplant waiting list, which, at the time of writing this manuscript, had less than 1,000 patients; fewer than 3,000 lung transplants are performed annually. The heart transplant waiting list had similar numbers, with approximately 3,500 patients awaiting transplantation.

\section{Conclusions}

The changes made by KAS have made remarkable strides in providing equity in access to transplants while also balancing utility. Recently implemented amendments to KAS that replaced 
the Donor Service Area distribution with 250 nautical mile circles may further increase transplant rates for those who are harder to match with due to biological reasons and those who are predicted to have high re-transplant rates, among other factors $[12,13,15,16]$. The clarity in acceptable antiA2 titers may also increase transplantation among the ABO-incompatible recipients. However, since even top-of-the-list priority for any compatible kidney donor in the nation was shown to be inadequate for boosting transplant access for the most highly sensitized kidney candidates, further allocation policy changes may offer little help to these patients. Several thousand candidates appear to have such an insurmountable immunological challenge that no compatible deceased kidney donor seems to exist. Other modalities, including kidney paired donation and desensitization, either alone or in combination, are likelyrequired to provide a realistic chance of transplantation for this highly disadvantaged group [4].

\section{Author Contributions}

1. Participated in research design: Angela Q Maldonado, PharmD; Kristoffer Sjöholm, PhD; Joshua Lee, MD; Darren E Stewart, MS; 2. Participated in the writing of the paper: Angela Q Maldonado, PharmD; Kristoffer Sjöholm, PhD; Christian Kjellman, PhD; Håkan Olsson, PhD; Darren E Stewart, MS; 3. Participated in the performance of the research: Angela $Q$ Maldonado, PharmD; Kristoffer Sjöholm, PhD; Darren E Stewart, MS; 4. Contributed new reagents or analytic tools; 5. Participated in data analysis: Angela Q Maldonado, PharmD; Kristoffer Sjöholm, PhD; Darren E Stewart, MS.

\section{Funding}

This research was funded by Hansa Biopharma AB.

\section{Competing Interests}

The authors have declared that no competing interests exist.

\section{References}

1. Friedewald J, Samana CJ, Kasiske BL, Israni AK, Stewart D, Cherikh W, et al. The kidney allocation system. Surg Clin North Am. 2013; 93: 1395-1406.

2. Part 121-Organ producement and transplantation network [Internet]. Electronic Code of Federal Regulations; 2021. Available from: https://www.ecfr.gov/cgi-bin/textidx?SID=bb60e0a7222f4086a88c31211cac77d1\&mc=true\&node=pt42.1.121\&rgn=div5.

3. Nelson PW, Shield III CF, Muruve NA, Murillo D, Warady BA, Aeder MI, et al. Increased access to transplantation for blood group $B$ cadaveric waiting list candidates by using $A_{2}$ kidneys: Time for a new national system? Am J Transplant. 2002; 2: 94-99.

4. Jackson KR, Covarrubias K, Holscher CM, Luo X, Chen J, Massie AB, et al. The national landscape of deceased donor kidney transplantation for the highly sensitized: Transplant rates, waitlist mortality, and posttransplant survival under KAS. Am J Transplant. 2018; 4: 1129-1138.

5. Schinstock CA, Smith BH, Mongtomery RA, Jordan SC, Bentall AJ, Mai M, et al. Managing highly sensitized renal transplant candidates in the era of kidney paired donation and the new kidney allocation system: Is there still a role for desensitization? Clin Transplant. 2019; 33: e13751. 
6. Jackson KR, Motter JD, Kernodle A, Desai N, Thomas AG, Massie AB, et al. How do highly sensitized patients get kidney transplants in the United States? Trends over the last decade. Am J Transplant. 2020; 20: 2101-2112.

7. Bryan $C F$, Cherikh WS, Sesok-Pizzini $D A . A_{2} / A_{2} B$ to $B$ renal transplantation: Past, present, and future directions. Am J Transplant. 2016; 16: 11-20.

8. Williams WW, Cherikh WS, Young CJ, Fan PY, Cheng Y, Distant DA, et al. First report on the OPTN national variance: Allocation of $A_{2} / A_{2} B$ deceased donor kidneys to blood group $B$ increases minority transplantation. Am J Transplant. 2015; 15: 3134-3142.

9. Azzi $Y$, Nair G, Loarte-Campos P, Ajaimy M, Graham J, Liriano-Ward L, et al. A safe anti-A 2 titer for a successful $A_{2}$ incompatible kidney transplantation: A single-center experience and review of the literature. Transplant Direct. 2021; 7: e662.

10. Stewart DE, Kucheryavaya AY, Klassen DR, Turgeon NA, Formica RN, Aeder MI. Changes in deceased donor kidney transplantation one year after KAS implementation. Am J Transplant. 2016; 16: 1834-1847.

11. Hahn AB, Mackey M, Constantino D, Ata A, Chandolias N, Lopez-Soler R, et al. The new kidney allocation system does not equally advantage all very high CPRA candidates - A single center analysis. Hum Immunol. 2017; 78: 37-40.

12. Stewart DE, Wilk AR, Toll AE, Harper AM, Lehman RR, Robinson AM, et al. Measuring and monitoring equity in access to deceased donor kidney transplantation. Am J Transplant. 2018; 18: 1924-1935.

13. Stewart D, Wilk A, Klassen DK. KAS turns four: The state of deceased donor kidney allocation in the U.S. OBM Transplant. 2018; 3: 1.

14. Martins PN, Mustian MN, MacLennan PA, Ortiz JA, Akoad M, Caicedo CJ, et al. Impact of the new kidney allocation system $A_{2} / A_{2} B \rightarrow B$ policy on access to transplantation among minority candidates. Am J Transplant. 2018; 18: 1947-1953.

15. Kidney Allocation System [Internet]. Available from: https://optn.transplant.hrsa.gov/learn/professional-education/kidney-allocation-system/.

16. Analysis report: Update [Internet]. [cited date 2021 February 01]. Available from: https://optn.transplant.hrsa.gov/media/2985/ki2019 01 analysisreport.pdf.

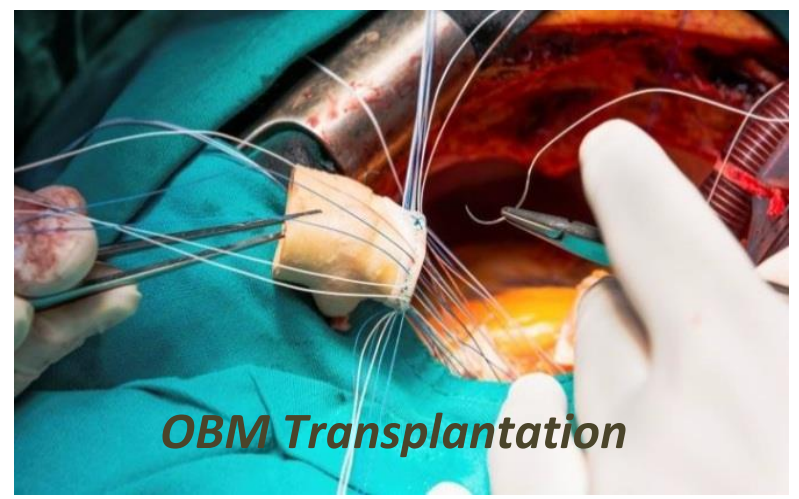

Enjoy OBM Transplantation by:

1. Submitting a manuscript

2. Joining in volunteer reviewer bank

3. Joining Editorial Board

4. Guest editing a special issue

For more details, please visit: http://www.lidsen.com/journals/transplantation 